\title{
A Spectroscopic and structural study of FeCoSb alloy
}

\author{
Saba J. Kadhem \\ Department of Physics, College of Science, University of Baghdad, Iraq \\ E-mail: saba_kadhem@yahoo.com
}

\begin{abstract}
$\mathrm{Fe}, \mathrm{Co}$ and $\mathrm{Sb}$ nanopowders were fruitfully prepared by electrical wire explosion method in Double distilled and de-ionized water (DDDW) media. The formation of iron, cobalt and antimony (FeCoSb) alloy nanopowder was monitored by X-ray diffraction. The $\mathrm{x}$-ray diffraction pattern indicates that there are iron, cobalt and antimony peaks. Optical properties of this alloy nanoparticles were characterized by UV-Visible absorption spectra. The absorption peak position is shifted to the lower wavelengths when the current increases. That means the mean size of the nanoparticles controlled by changing the magnitude of the current. The surface morphological analysis is carried out by employing Scanning Electron Microscope (SEM). Particles with varies size were observed also from the images the some particles have uneven shapes with agglomerate and the other have spherical shape. The exploding $\mathrm{FeCoSb}$ alloy wire plasma parameters is study by optical emission spectroscopy. The emission spectra of the plasma have been recorded and analyzed. The plasma electron temperature $\left(\mathrm{T}_{\mathrm{e}}\right)$, was determined by Boltzmann plot, and the electron density (ne), by Stark broadening for wire with diameter $0.3 \mathrm{~mm}$ and current of $75 \mathrm{~A}$ in distilled water.
\end{abstract} Key words

$\mathrm{Fe}$ alloy, $\mathrm{FeCoSb}$ alloy, exploding wire, nanoparticles, optical properties

\author{
Article info. \\ Received: Nov. 2018 \\ Accepted: Mar. 2019 \\ Published: Jun. 2019
}

\section{FeCoSb دراسة طيفية وتركيبية لسبيكة \\ صبا جواد كاظم \\ قسم الفيزياء، كلية العلوم ، جامعة بخداد، العراق}

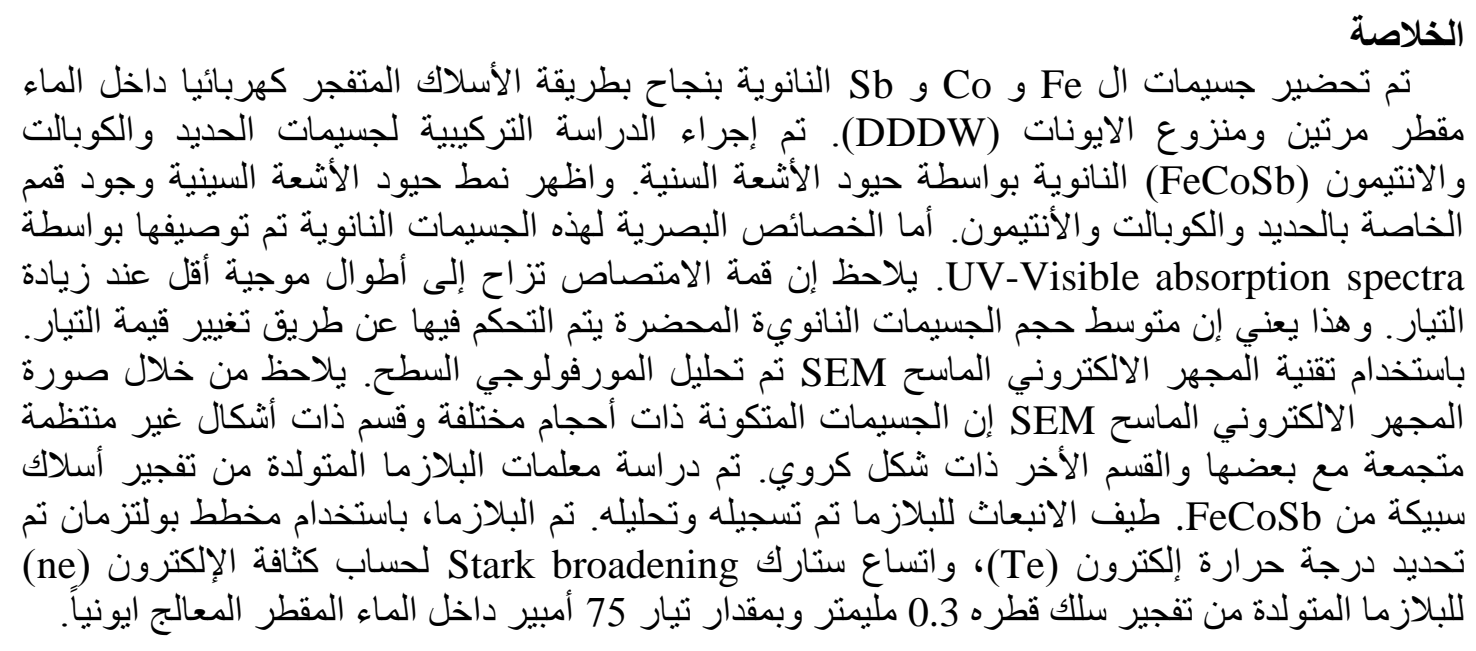




\section{Introduction}

Nanoparticles have been a source of enormous attention because of their novel electrical, optical, physical, chemical and magnetic properties. There are a many of reports of physical and chemical processes for fabrication of nanoparticles one of them is by using plasma (exploding wire method) [1]. The nanoparticles have momentous possible for a wide range of applications like composite materials, catalysis, magnetic recording media, magnetic fluids, optoelectronic materials, fuel cells and sensors [2, 3]. FeCoSb alloys are attracting greatly attention because their extraordinary physical, chemical, and magnetic properties. Recently, $\mathrm{FeCoSb}$ alloy nanoparticles have unique properties that be different from those of the bulk materials [4, 5]. These novel properties are because high surface to volume ratio, morphology, small particle size and surface coating of nanoparticles. $\mathrm{FeCoSb}$ alloy nanoparticles have interesting application like biomedical and catalytic applications [6].

The preparation methods influence the properties of nanoscale metal such as iron particles. There are chemical and physical methods are used to produce metal and metalloid nanoparticles in the form of powders and colloids [7, 8]. In the present study we report the characterization and unusual optical of $\mathrm{Fe}, \mathrm{Co}$ and $\mathrm{Sb}$ nanoparticles prepared from the $\mathrm{FeCoSb}$ alloy using the exploding wire technique. Physical-chemical description has considered X-ray diffraction (XRD), UV- visible and scanning electron microscopy (SEM). Also The plasma which produced by exploding wire method characteristics were investigated based on optical emission spectroscopy (OES).

\section{Experimental setup}

In this work, $\mathrm{FeCoSb}$ alloy wire with diameter of $0.3 \mathrm{~mm}$ was exploded in Double distilled and de-ionized water (DDDW). The explosion was carried out with three values of current 50,75 and $150 \mathrm{~mA}$. The source voltage available was 35 volts. Referring to Fig.1, a reaction beaker with volume $500 \mathrm{~mL}$ was used as the explosion chamber as shown in Fig.1. When the first electrode (wire) touched the second electrode (plate) the wire vaporized, and convert into plasma. The material for wire and plate is the same but with reverse polarity. The exploded wire is related to the perfect geometry during the wire guide. The plates were set on the base of reaction beaker which filled with $100 \mathrm{ml}$ of DDDW. The electrical circuit leftovers open until the contact is made by the wire on to the plate mechanically resulting in the wire explosion through a non linear process in very short time. The nanoparticles disperse in the DDDW solution.

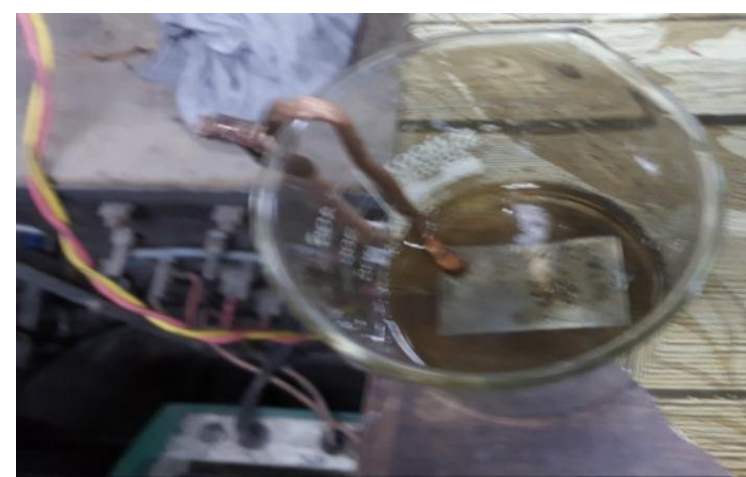

Fig.1: The system of explosion wire in liquid media. 
The wires have been exploded by bringing the alloy wire into speedy contact with the plate. The wire is exposed to several sparks and is being replaced after several contacts. The electrical explosion wire (EEW) process is very energy exhaustive since only relatively low voltages are applied, and also results in huge quantities of nanoparticles being formed from both the consumed electrodes. The experimental parameters are summarized in Table 1.

Table 1: Summary of experimental parameters.

\begin{tabular}{|c|c|}
\hline Wire Materials & FeCoSb alloy \\
\hline Wires diameter & $0.3 \mathrm{~mm}$ \\
\hline Applied current & $50-75$ and $150 \mathrm{~A}, \mathrm{DC}$ \\
\hline Wire length & $20 \mathrm{~cm}$ \\
\hline Metal plate & $(70 \times 40) \mathrm{mm}$ and thickness of $(2 \mathrm{~mm})$ \\
\hline Liquid type & DDDW (Double distilled and de-ionized water $)$ \\
\hline Liquid volume & $100 \mathrm{~mL}$ \\
\hline
\end{tabular}

Fig. 2 shows image of FeCoSb alloy colloid, prepared by electrical explosion wire technique, in DDDW media. We noted from the figure that

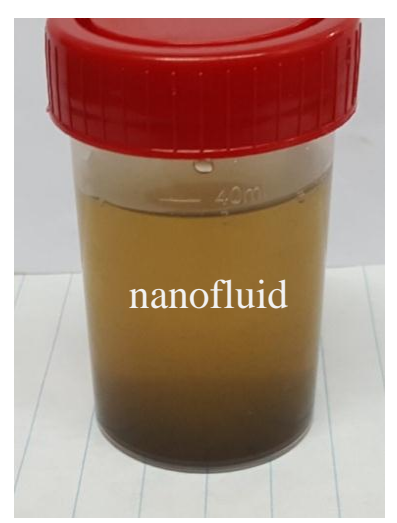

$\mathbf{a}$ the nanoparticles are attracted to the magnet. That mean the composit contains Irion nanoparticles.

Fig. 2: a) Image of FeCoSb alloy colloid prepared by EEW in DDDW. b) Image of FeCoSb nanoparticles which attracted to the magnet.

\section{UV-Visible absorption spectrum of Fe nanofluid}

Fig. 3 illustrated the absorbance of nanofluid that prepared from alloy wire at different current. It is clearly from this figure that the optical properties depend on the amount of current. The absorption peak position is shifted to the ultraviolet region when the current increased that means the diameters of the particles decreases with the current increases. 


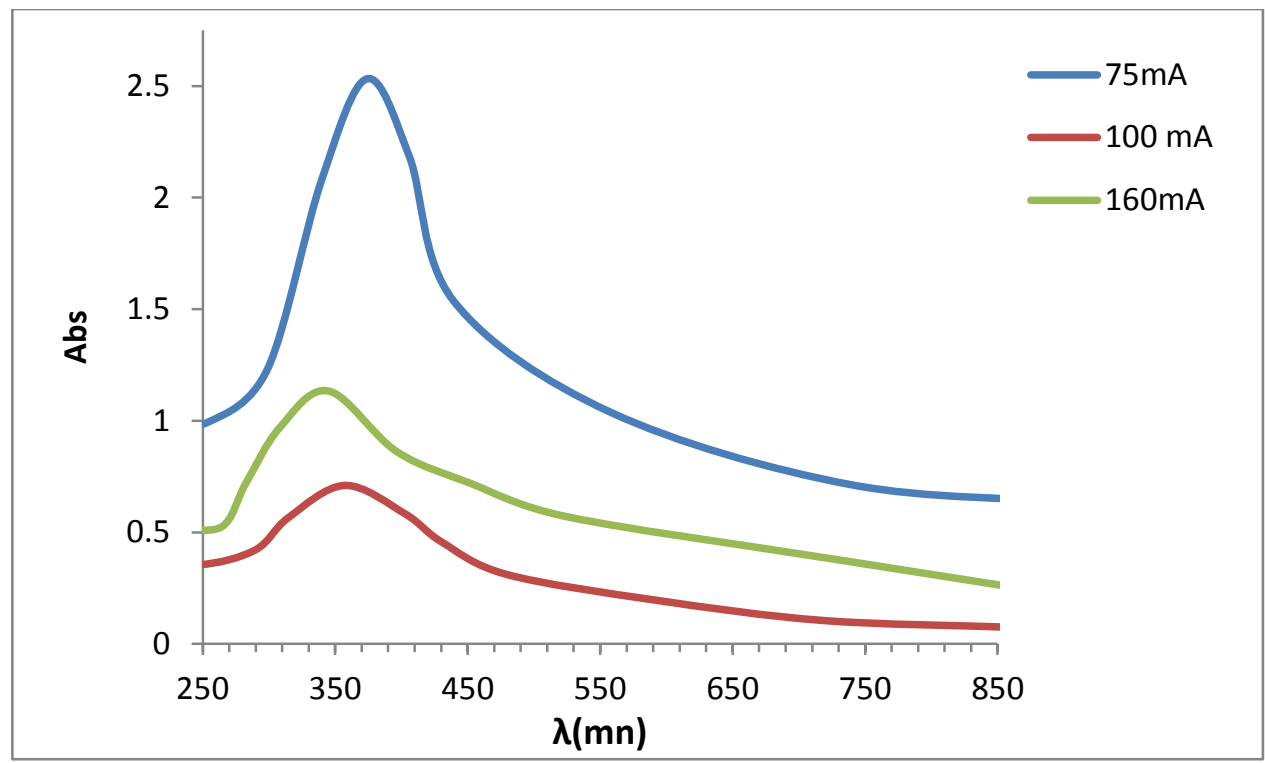

Fig. 3: Absorbance within UV visible for FeCoSb alloy nanofluid from alloy wire with $0.3 \mathrm{~mm}$ diameter in DDDW at three different values of current.

\section{X- ray diffraction (XRD)}

The crystal structure of the FeCoSb alloy-nanoparticles dispersed in DDDW is examined with X-Ray diffraction. Fig.4 shows the XRD pattern of the alloy nanoparticles dispersed in DDDW. The XRD pattern as a $2 \theta$ plot scanning from $20^{\circ}-80^{\circ}$ was recorded to determine crystallanity and structure. The peak obtained around $44.5^{\circ}$ corresponds to the (110) line of $\mathrm{Fe}$ (FCC). The peak position of the Fe nanoparticles planes (200) was at $66.16^{\circ}$ and it were shifted to slightly higher $2 \theta$ values. Another signals centered around $61^{\circ}$ is detected, thus including the presence of face- centered-cubic (FCC) cobalt nanoparticles corresponds to the (200) line. There are three peaks at $29.852^{\circ}$, $48.892^{\circ}$ and $55.871^{\circ}$ characteristic of (220), (100) and (101) diffraction plan of hexagonal centered cobalt. The peaks centered at $21.3^{\circ}$ and $35.213^{\circ}$ corresponds to the (003) and (014) line of $\mathrm{Sb}$.

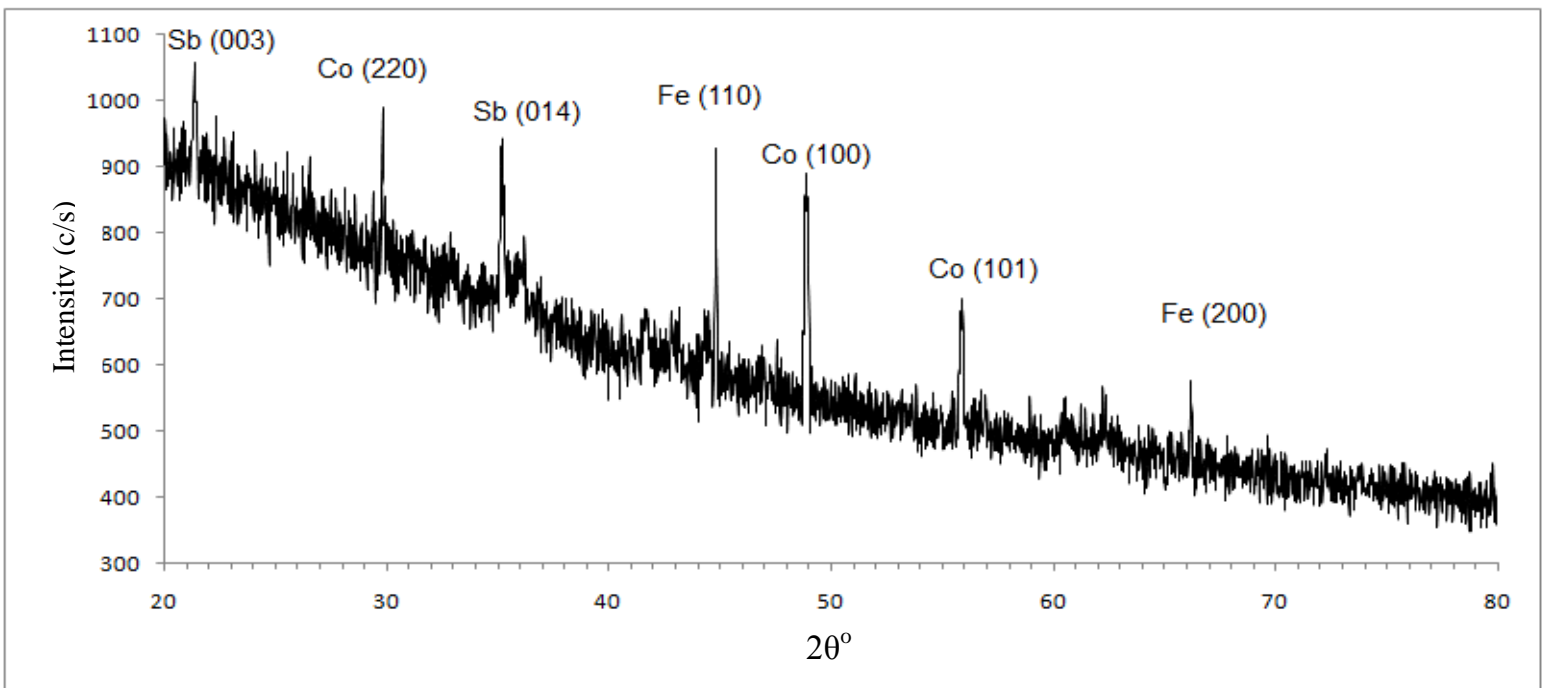

Fig. 4: X-ray diffraction pattern for FeCoSb alloy nanoparticles that obtain by exploded wire method with different diameter in DDDW media. 


\section{Scanning Electron Microscopic (SEM)}

Fundamental study of samples morphology was obtained using particular electron microscopy. It is possible to observe the external structures of nanoparticles using SEM, and it is performed to the characterize of the texture of the deposited metal as a thin film on substrate. From the images there are varies size are observed also from the images the some particles have irregular shapes with agglomerate and the other have spherical shape as shown in Fig.5.
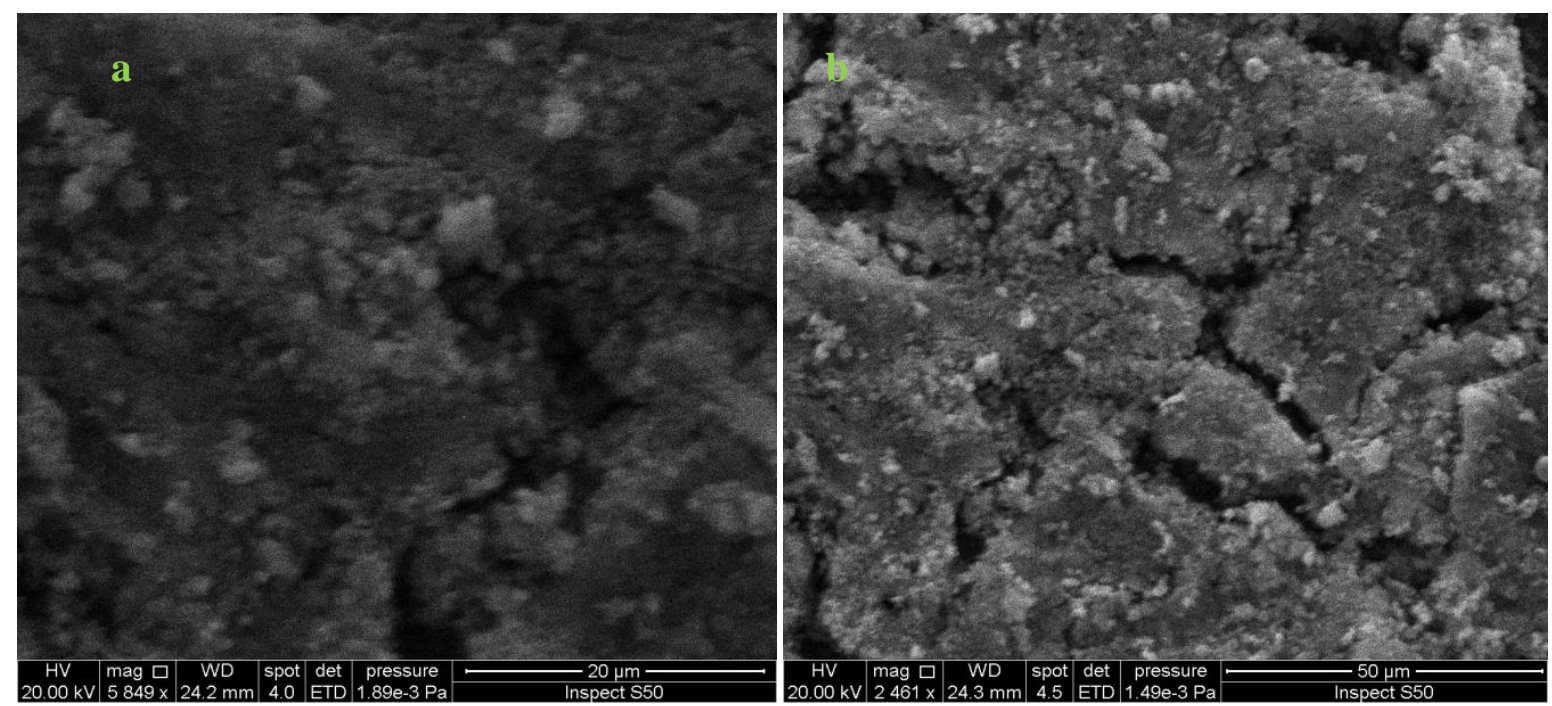

Fig. 5: SEM images for synthesized alloy NPS in water, obtained from $0.3 \mathrm{~mm}$ exploded FeCoSb wire at; a) high magnification, b) low magnification.

Energy dispersive $\mathbf{X}$-ray analysis

The elemental analysis of the alloy is investigation by Energy Dispersive $\mathrm{X}$-ray spectroscopy (EDX) analysis as shown in Fig. 6. EDX measurement confirms the existence of the alloy in the film. From Fig. 6, there are many strong peaks for iron, cobalt and antimony but the strongest peak is to iron. The weak peak of $\mathrm{Si}$ and $\mathrm{Ca}$ shown in the EDX spectra correspond to the glass substrate material.

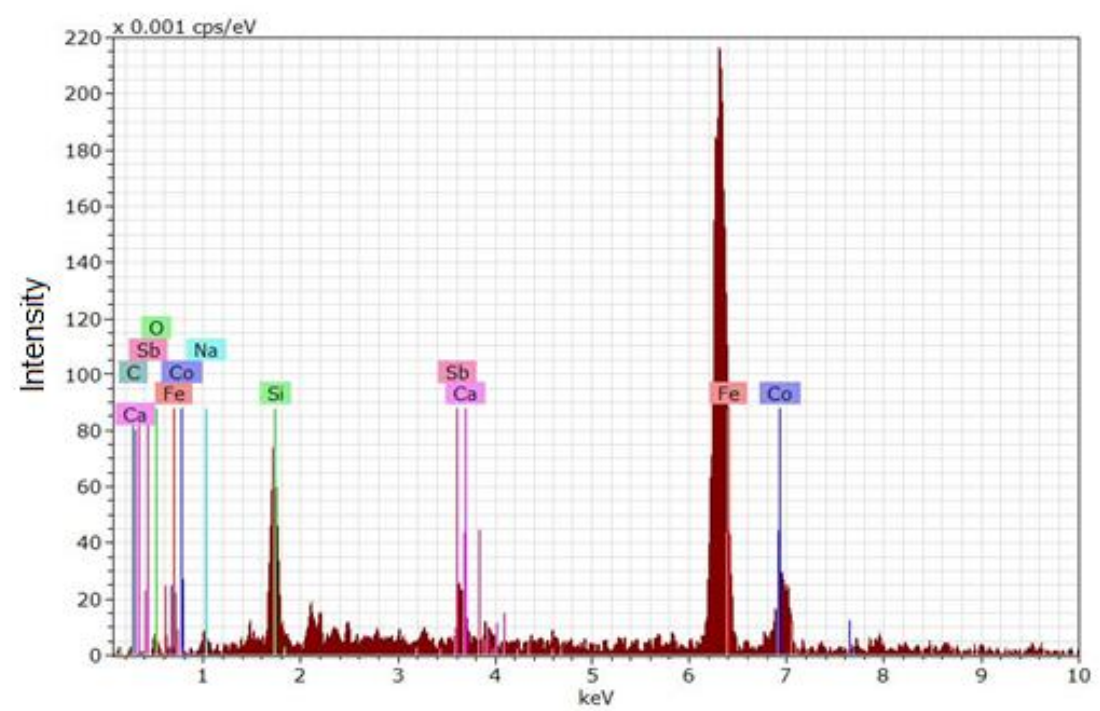

Fig. 6: The EDX energy of the alloy nanoparticles. 


\section{The spectroscopic analysis}

Fig.7 shows the optical emission spectrum emitted from the Plasma produced by exploding $\mathrm{FeCoSb}$ alloy wire with diameter of $0.3 \mathrm{~mm}$ and current 75A. The emission spectra of this alloy plasma have been recorded and analyzed. The plasma electron temperature (Te), was calculated by using Boltzman plot method, and the electron density (ne) was determine by Stark broadening using seven Fe I line at 516.74, 517.15, 522.71, 532.85, $537.1,537.14$ and $540.4 \mathrm{~nm}$ for the alloy wire. Also peaks at 217.581 and $556.812 \mathrm{~nm}$ correspond to antimony (Sb I) and (Sb II) respectively. Small peaks at 258.326 and $345.351 \mathrm{~nm}$ correspond to Co I and Co II respectively. These elements come from the FeCoSb alloy. Also some of ionic oxygen lines are founded in the inset figure. The $T_{e}$ values were deduced from reveres of best fitting line for the relation between $\ln \left(\frac{I_{j i} \lambda_{j i}}{g_{j} A_{j i}}\right)$ versus upper energy level $\left(E_{j}\right)$. From Fig.8, it can be noticed that the peak located at $540.3 \mathrm{~nm}$ corresponding to Fe line for iron atoms produced from the alloy molecular dissociate.

The Fe I line was used to calculate the electron temperature. The $\mathrm{T}_{\mathrm{e}}$ value can be calculated using Fig.9 from reveres of best fitting line. The constants Values for the iron emission lines taken from reference [9]. The equations of fitting lines and the $R^{2}$ were shown in the figure. $R^{2}$ is the statistical coefficient indicating the quality of the linear fit.

Table 2 shows the diameter of wire, the calculated electron temperature $T_{e}$, electron density $\left(\mathrm{n}_{\mathrm{e}}\right)$, plasma frequency $\left(f_{p}\right)$ and Debye length $\left(\lambda_{D}\right)$ for FeCoSb alloy plasma produced by exploding wire for $0.3 \mathrm{~mm}$ wire diameters and $75 \mathrm{~A}$ current.

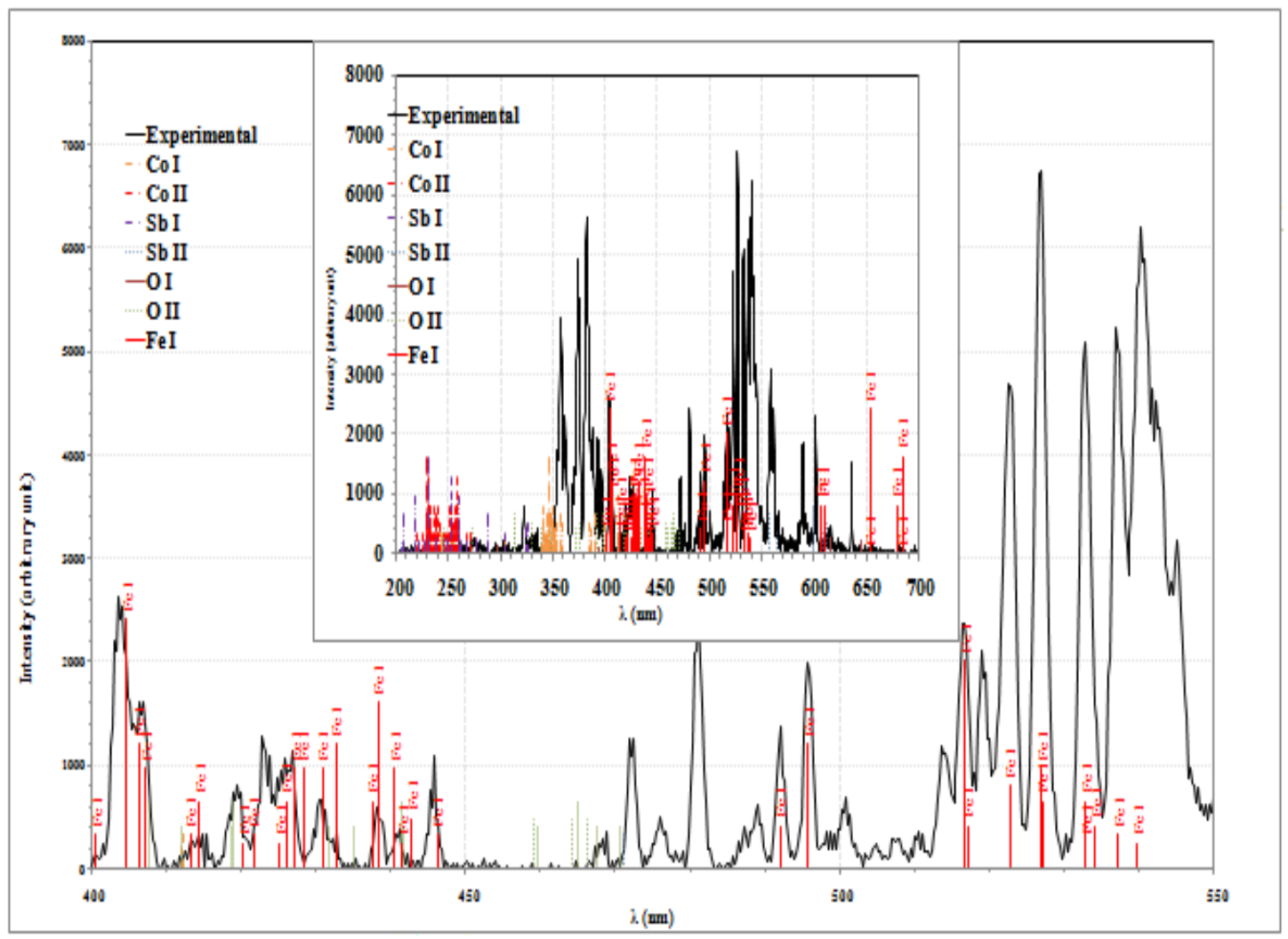

Fig. 7: Emission spectra for FeCoSb alloy exploding wire. 


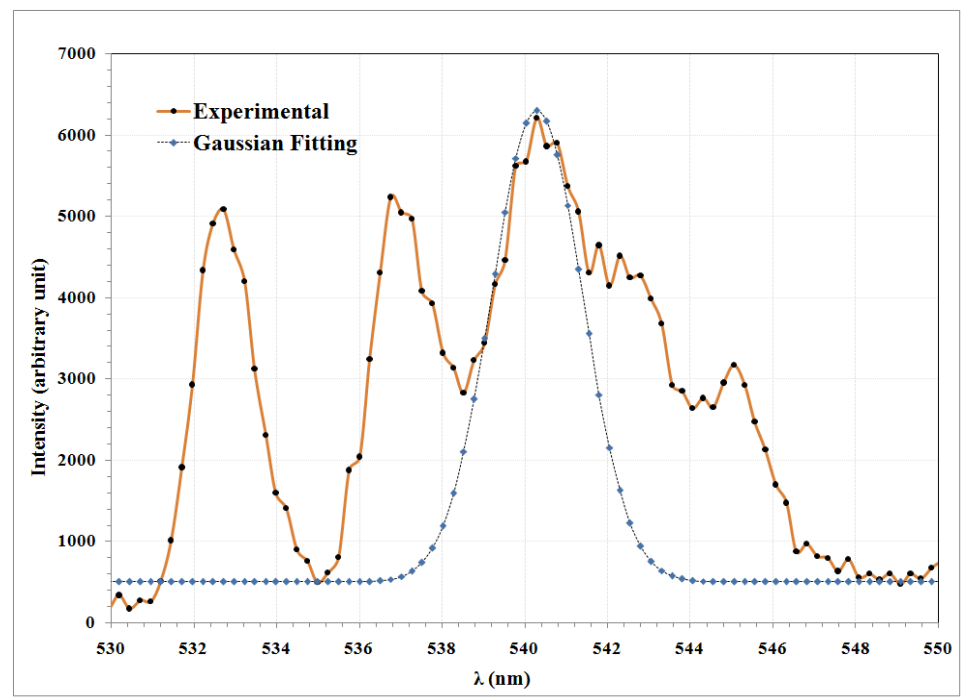

Fig. 8: Fe I peaks at $540.4 \mathrm{~nm}$ broadening and there Gaussian fitting.

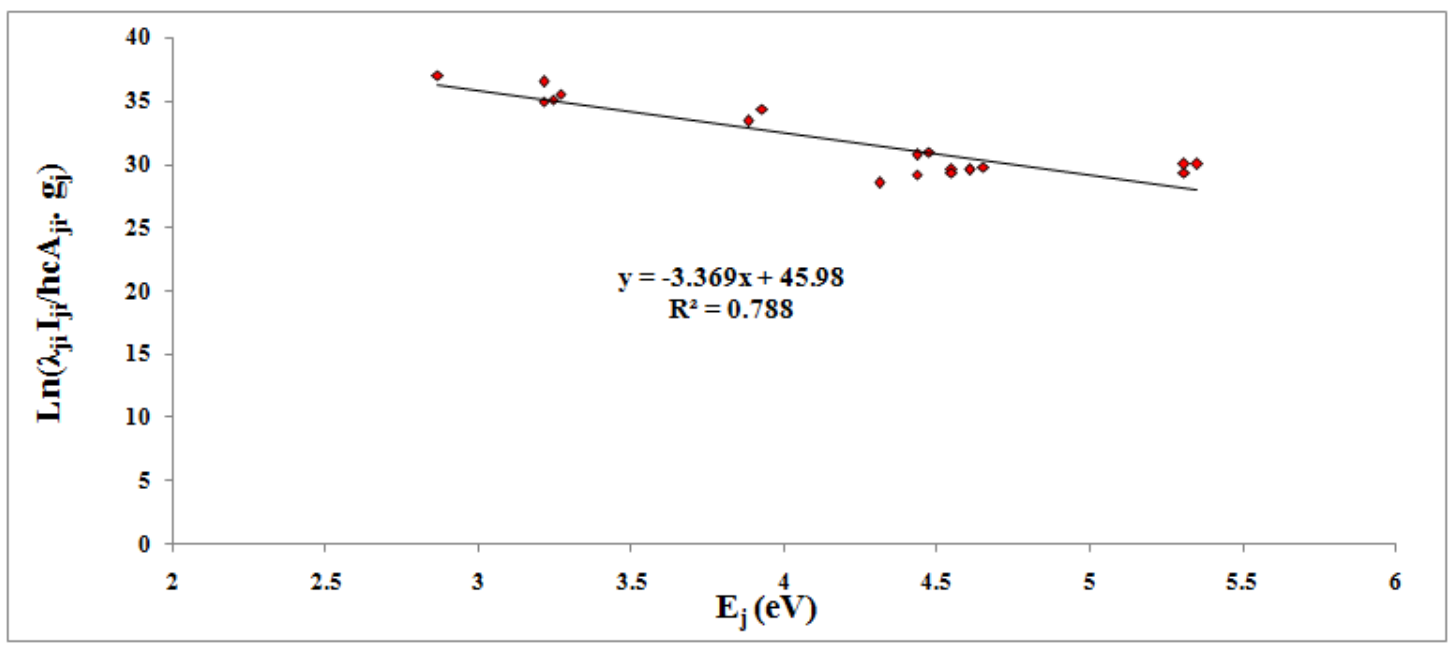

Fig. 9: Boltzmann plot from the Fe I lines produced by exploding wire.

Table 2: Plasma parameters calculated from emission spectrum lines emitted from FeCoSb alloy plasma produced by exploding wire.

\begin{tabular}{|c|c|}
\hline Wire diameter & $0.3 \mathrm{~mm}$ \\
\hline \hline electron temperature $\mathrm{T}_{\mathrm{e}}$ & $0.300 \mathrm{eV}$ \\
\hline \hline FWHM & $2.60 \mathrm{~nm}$ \\
\hline electron density $\left(\mathrm{n}_{\mathrm{e}}\right)$ & $1.44 * 10^{18} \mathrm{~cm}^{-3}$ \\
\hline \hline plasma frequency $\left(\mathrm{f}_{\mathrm{p}}\right)$ & $1.1^{*} 10^{13} \mathrm{~Hz}$ \\
\hline \hline Debye length $\left(\lambda_{\mathrm{D}}\right)$ & $3.4^{-6} 10^{-6} \mathrm{~cm}$ \\
\hline \hline The number of particles in a Debye sphere $\mathrm{N}_{\mathrm{d}}$ & $0.235^{*} 10^{3}$ \\
\hline Plasma ideality & 0.874 \\
\hline
\end{tabular}

The $540.4 \mathrm{~nm}$ iron line peak shows in Fig. 8. The full width at half maximum was found by Gaussian fitting. From the measured width which depending on Stark effect and the standard line width for this line. The electrons density were calculated by the equation $[8,9]$ : 


$$
\lambda_{D}=\left(\frac{k_{\beta} T_{e}}{4 \pi e^{2} n_{0 e}}\right)^{1 / 2}
$$

Plasma frequency which given by [10]:

$$
\omega_{p}=\sqrt{\frac{N_{e} q_{e}^{2}}{\epsilon_{o} m_{e}}}
$$

Also the number of particles $\left(\mathrm{N}_{\mathrm{D}}\right)$ in a Debye sphere [10]:

$N_{D}=n_{e}\left[\frac{4 \pi \lambda_{D}^{3}}{3}\right]=\frac{1.38 \times 10^{6} T_{e}^{3 / 2}}{n_{e}^{1 / 2}}$

where: $\mathrm{T}_{\mathrm{e}}$ in $\mathrm{K}$.

\section{Conclusions}

Metal and metalloid nanoparticles can be produced by one step simple, effective and low cost technique that is the Electrical Explosion Wire (EEW) technique and it may be applied in air and liquid. Mean particle size could be controlled by proper selection of the current, and breakdown strength of the medium. The peaks properties as peak position and peak intensity are reliable indicators for identifying the size and concentration. SEM image gives good information about the shape, size and the size distribution for the nanoparticles.

The nanoparticles formed from the wire have spherical shapes with agglomerate. $\mathrm{Fe}, \mathrm{Co}$, and $\mathrm{Sb}$ components in the alloy nanopowders are uniformly dispersed. X-ray examination included the peaks of the three elements in the alloy.

\section{References}

[1] A. Alqudami and S. Annapoorni, Plasmonics, 2 (2007) 5-13.

[2] P. Sen, J. Ghosh, A. Abdullah, P. Kumar, Vandana, Proc. Indian Acad. Sci. (Chem. Sci.), 115, 5 \& 6 (2003) 499-508.

[3] J.Y. Yun, H. M. Lee, S.Y. Choi, S. S. Yang, D. W. Lee1, Y. J. Kim, B. K.Kim Materials Transactions, 52, 2 (2011) 250-253.

[4] L. H. Bac, B. K. Kim, J. S. Kim, J. C. Kim, Journal of Magnetics, 16, 4 (2011) 435-439.

[5] D. Carta, G. Mountjoy, M. Gass, G. Navarra, M. F. Casula, A. Corrias, The Journal of Chemical Physics, 127 (2007) 204705-1_204705-9.

[6] S. Dadashi, R. Poursalehi H. Delavari, Procedia Materials Science, 11 (2015) 722-726.

[7] T. Kulik, A. Wlazlowska, J. Ferenc, J. Latuch, IEEE Transactions on Magnetics, 38, 5, (2002) 30753077.

[8] M. Y. Tafti, M. Saleemi, M. S. Toprak, M. Johnsson, A. Jacquot, M. Jägle, M. Muhammed, Open Chem., 13 (2015) 629-635.

[9] R. Voda, A. Negrea, L. Lupa, M. Ciopec, P. Negrea, C. M. Davidescu, M. Butnariu, Open Chem., 13 (2015) 743-747.

[10] A. A-K. Hussain, A. Abd AlRazzaq, Iraqi Journal of Physics, 14, 31 (2016) 205-214. 